\title{
Methylene blue exerts a neuroprotective effect against traumatic brain injury by promoting autophagy and inhibiting microglial activation
}

\author{
MINGFEI ZHAO ${ }^{1 *}$, FENG LIANG $^{1 *}$, HANGDI XU $^{2}$, WEI YAN $^{1}$ and JIANMIN ZHANG ${ }^{1}$ \\ ${ }^{1}$ Department of Neurosurgery, Second Affiliated Hospital, School of Medicine, Zhejiang University, \\ Hangzhou, Zhejiang 310009; ${ }^{2}$ Department of Respiratory Medicine, Sir Run Run Shaw Hospital, \\ School of Medicine, Zhejiang University, Hangzhou, Zhejiang 310016, P.R. China
}

Received September 27, 2014; Accepted May 21, 2015

DOI: $10.3892 / \mathrm{mmr} .2015 .4551$

\begin{abstract}
Traumatic brain injury (TBI) leads to permanent neurological impairment, and methylene blue (MB) exerts central nervous system neuroprotective effects. However, only one previous study has investigated the effectiveness of $\mathrm{MB}$ in a controlled cortical impact injury model of TBI. In addition, the specific mechanisms underlying the effect of $\mathrm{MB}$ against TBI remain to be elucidated. Therefore, the present study investigated the neuroprotective effect of MB on TBI and the possible mechanisms involved. In a mouse model of TBI, the animals were randomly divided into sham, vehicle (normal saline) or MB groups. The treatment time-points were 24 and $72 \mathrm{~h}$ (acute phase of TBI), and 14 days (chronic phase of TBI) post-TBI. The brain water content (BWC), and levels of neuronal death, and autophagy were determined during
\end{abstract}

Correspondence to: Dr Wei Yan or Professor Jianmin Zhang, Department of Neurosurgery, Second Affiliated Hospital, School of Medicine, Zhejiang University, 88 Jiefang Road, Hangzhou, Zhejiang 310009, P.R. China

E-mail: ywisyw@163.com

E-mail: zjm135@vip.sina.com

Abbreviations: ALS, amyotrophic lateral sclerosis; BBB, blood-brain barrier; BWC, brain water content; CCI, controlled cortical impact; cGMP, cyclic guanidine monophosphate; $\mathrm{dw}$, dry weight; HE, hematoxylin-eosin; HRP, horseradish peroxidase; Iba-1, ionized calcium-binding adapter molecule 1; IgG, immunoglobulin G; LC3, microtubule-associated protein light chain 3; MB, methylene blue; mNSS, modified neurological severity score; NeuN, neuronal nuclear antigen; NIH, National Institutes of Health; NO, nitric oxide; NOS, NO synthase; PBS, phosphate-buffered saline; sGC, soluble guanylate cyclase; SOD1G93A, superoxide dismutase 1 gene mutation G93A; TBI, traumatic brain injury; US FDA, United States Food and Drug Administration; ww, wet weight

Key words: methylene blue, traumatic brain injury, autophagy, microglia the acute phase, and neurological deficit, injury volume and microglial activation were assessed at all time-points. The injured hemisphere BWC was significantly increased $24 \mathrm{~h}$ post-TBI, and this was attenuated following treatment with MB. There was a significantly higher number of surviving neurons in the MB group, compared with the Vehicle group at 24 and $72 \mathrm{~h}$ post-TBI. In the acute phase, the MB-treated animals exhibited significantly upregulated expression of Beclin 1 and increased LC3-II to LC3-I ratios, compared with the vehicle group, indicating an increased rate of autophagy. Neurological functional deficits, measured using the modified neurological severity score, were significantly lower in the acute phase in the MB-treated animals and cerebral lesion volumes in the MB-treated animals were significantly lower, compared with the other groups at all time-points. Microglia were activated $24 \mathrm{~h}$ after TBI, peaked at $72 \mathrm{~h}$ and persisted until 14 days after TBI. Although the number of Iba-1-positive cells in the vehicle and MB groups $24 \mathrm{~h}$ post-TBI were not significantly different, marked microglial inhibition was observed in the MB group $72 \mathrm{~h}$ and 14 days after -TBI. These results indicated that $\mathrm{MB}$ exerts a neuroprotective effect by increasing autophagy, decreasing brain edema and inhibiting microglial activation.

\section{Introduction}

It is estimated that $>10,000,000$ individuals suffer traumatic brain injury (TBI) annually worldwide (1). TBI contributes to significantly to mortality rates, and several survivors are subject to complications, including neurological deficit and motor dysfunction (2).

Although survival rates following head injury have significantly improved in the last few decades due to advances in emergency and intensive care medicine, no effective proactive treatment options exist for augmenting functional recovery or for preventing long-term neuropsychiatric complications post-TBI (3). An increasing number of studies have demonstrated that the extent of brain damage post-TBI depends, not only on the initial trauma, but also on secondary injury caused by neuroinflammation, cerebral edema and breakdown of the blood-brain barrier (4). Secondary brain 
injury occurs between a duration of a few hours and several months, ultimately leading to neuronal death or neurodegeneration (2). Due these consequences, investigations have focused on the mechanisms or pathways of secondary brain injury to attempt to identify novels drugs for its inhibition or attenuation.

Methylene blue (MB) is an inexpensive, United States Food and Drug Administration-approved drug, which has been used safely in humans for $>120$ years (5). It is used for diagnostic procedures and for the treatment of methemoglobinemia, malaria, cyanide poisoning, carbon monoxide poisoning (6), cardiopulmonary bypass (7) and septic shock (8).

Previous studies have demonstrated that MB also exerts beneficial effects on the central nervous system. For example, it may exert neuroprotective activity against Parkinson's disease (9), amyotrophic lateral sclerosis (10) and ischemic stroke (11). A previous study investigated the effectiveness of $\mathrm{MB}$ in a rat model of controlled cortical impact (CCI) injury and revealed that $\mathrm{MB}$ minimized lesion volume, behavioral deficits and neuronal degeneration (12). However, the specific mechanisms of MB against TBI remain to be elucidated. Therefore, the present study investigated the neuroprotective effect of MB on TBI and the possible mechanisms involved, focusing on the association between $\mathrm{MB}$ and the mechanisms of secondary brain injury, including neuroinflammation, brain edema and autophagy.

\section{Materials and methods}

Animals. Mature male Institute for Cancer Research mice (25-30 g; Zhejiang Academy of Medical Sciences, Hangzhou, China) were used in the present study. A total of 210 mice were used in total (70/group). The animals were maintained on a $12 \mathrm{~h}$ light/dark cycle with access to food and water ad libitum. The study was approved by the Ethics Committee of Zhejiang University (Hangzhou, China), and all experimental procedures were approved by Zhejiang University Institutional Animal Care and Use Committee and conformed to the National Institutes of Health (NIH) Guide for the Care and Use of Laboratory Animals (13).

Model of TBI. The procedure used for creating the CCI model of TBI was as described previously (14). Briefly, the mice were anesthetized with pentobarbital sodium $(50 \mathrm{mg} / \mathrm{kg}$ intraperitoneally) and mounted in a stereotaxic frame. Following a midline incision and retraction of the skin, a $4 \mathrm{~mm}$ diameter craniotomy was performed over the right frontoparietal cortex using a $5 \mathrm{~mm}$ trephine, between the bregma and lambda, without damaging the underlying dura. The bone flap was removed and the CCI was produced perpendicular to the brain surface $\left(12^{\circ}\right.$ from the vertical) using a Benchmark CCI Stereotaxic Impactor (Benchmark Deluxe ${ }^{\mathrm{TM}}$; myNeuroLab, St. Louis, MO, USA) fitted with a $2.5 \mathrm{~mm}$ tip $(3.0 \mathrm{~m} / \mathrm{sec} ; 100 \mathrm{~ms}$ dwell time; $2 \mathrm{~mm}$ depth). The same surgeon performed all CCI surgeries for each cohort of mice. The core body temperature of the mice was maintained at $36.5-37^{\circ} \mathrm{C}$ during surgery using a rectal thermometer coupled to a heating pad. The bone flap was immediately replaced and sealed, and the scalp was sutured. The mice in the sham group underwent identical surgical procedures, but without CCI. All animals were returned to their cages when fully recovered from anesthesia in a heated chamber.

Experimental design. The mice were randomly assigned into either sham, vehicle (normal saline) or MB groups. Either normal saline or MB (1 mg/kg; 10mg/ml, cat. no. 0517-030110, American Regent, Inc. Shirley, NY, USA) was infused intraperitoneally for $1 \mathrm{~h}$ following TBI, and at $0.5 \mathrm{mg} / \mathrm{kg} 6 \mathrm{~h}$ following TBI. A total of $1 \mathrm{mg} / \mathrm{kg} \mathrm{MB}$ or the same volume of saline was administered daily for the next 3 days. The MB dosage and treatment time-points were determined based on a previous study (15) and preliminary experiments.

Evaluation of neurological deficits. The modified neurological severity score (mNSS) was used to examine the effects of $\mathrm{MB}$ on neurological deficits at all time-points following TBI. Neurological function was graded on a scale of $0-18$, as previously described (Table I) (16).

Brain water content (BWC). At $24 \mathrm{~h}$ and $72 \mathrm{~h}$ post-TBI, the mice were sacrificed and the brains were removed and divided into right and left hemispheres, cerebellum and brainstem, which were weighed immediately to obtain the wet weight (ww). The samples were dried at $80^{\circ} \mathrm{C}$ for $72 \mathrm{~h}$, following which the dry weight (dw) was measured. The BWC was calculated as follows: (ww - dw) / ww x 100\% (17).

Hematoxylin and eosin (HE) staining and lesion volume assessment. The mice were anesthetized with pentobarbital sodium $(50 \mathrm{mg} / \mathrm{kg}$ intraperitoneally; 1\%; Sigma-Aldrich, St. Louis, MO, USA), and then sacrificed by spinal dislocation at $24 \mathrm{~h}, 72 \mathrm{~h}$, and 14 days post-TBI for HE staining (Sigma-Aldrich) and immunohistochemistry. Briefly, the mice were deeply anesthetized, as described above, and were transcardially perfused with $20 \mathrm{ml}$ saline solution $\left(4^{\circ} \mathrm{C}\right)$, followed by $100 \mathrm{ml} 10 \%$ formalin phosphate-buffered saline solution (pH 7.4) for 30 min (Wuhan Jiahuayuan Chemical Co. Ltd., Wuhan, China). The brains were then removed and post-fixed in the same perfusate at $4^{\circ} \mathrm{C}$ for $72 \mathrm{~h}$, sectioned coronally from $1 \mathrm{~mm}$ anterior to the lesion site to the posterior margin of the lesion, and paraffin-embedded (Sigma-Aldrich). The area of the lesion was determined in 10 sections at $300 \mu \mathrm{m}$ intervals throughout the entire lesion ( $4 \mu \mathrm{m}$ thickness per section). The sections were HE-stained using standard methods $(0.5 \%$ hematoxylin for $5 \mathrm{~min}$ at $25^{\circ} \mathrm{C} ; 0.5 \%$ eosin for $1 \mathrm{~min}$ at $25^{\circ} \mathrm{C}$ ), imaged with a stereomicroscope using bright-field illumination (Olympus BX51; Olympus, Tokyo, Japan) and analyzed using ImageTool software 3.0 (University of Texas Health Science Center, Texas, TX, USA). The brain injury volume $\left(\mathrm{mm}^{3}\right)$ was calculated as follows: $\Sigma(\mathrm{An}+\mathrm{An}+1) \mathrm{x} \mathrm{d} / 2$, where $A$ is the area of apparent brain tissue loss and $d$ is the distance between sections, as previously described $(14,18)$, with minor modifications.

Immunohistochemistry. The samples were treated, as described above. Coronal sections ( $4 \mu \mathrm{m}$ thick) were obtained using a microtome (Leica CM1900; Lyca Microsystems $\mathrm{GmbH}$, Wetzlar, Germany) and heated in an oven at $60^{\circ} \mathrm{C}$ overnight for deparaffinization. Antigen retrieval was 
Table I. Modified neurological severity scores (16).

Assessment

Score

Motor assessments

Raising mice by tail (normal, 0; maximum, 3)

Flexion of forelimb

Flexion of hindlimb

Head movement $>10^{\circ}$ to vertical axis within $30 \mathrm{sec}$

Putting mice on the floor (normal, 0 ; maximum, 3)

Normal walk

Unable to walk straight

Circling toward paretic side

Falls down to paretic side

Sensory assessments

Placing test (visual and tactile test)

Proprioceptive test (deep sensation, pushing paw against table edge to stimulate limb muscles)

Beam balance test (normal, 0; maximum, 6)

Balances with steady posture

Grasps side of beam

Hugs beam but one limb falls from beam

Hugs beam and two limbs fall from beam, or spins on beam (>60 sec)

Attempts to balance on beam but falls off ( $>40 \mathrm{sec})$

Attempts to balance on beam but falls off ( $>20 \mathrm{sec})$

Falls off; no attempt to balance or hang on to beam within $20 \mathrm{sec}$

Reflex absence and abnormal movements (normal, 0; maximum, 4)

Pinna reflex (head shake when auditory meatus is touched)

Corneal reflex (eye blink when cornea is lightly touched with cotton)

Startle reflex (motor response to a brief knocking noise)

Seizures, myoclonus, myodystony

Maximum points 18 . One point is awarded for the inability to perform a tasks or for lack of a tested reflex (pinna reflex, corneal reflex, startle reflex). 13-18, severe injury; 7-12, moderate injury; 1-6, mild injury.

performed in citrate buffer $(\mathrm{pH} 6.0 ; 0.01 \mathrm{mmol} / \mathrm{L})$ containing citric acid (cat. no. 791725) and sodium citrate tribasic dihydrate (cat. no. 6132-04-3; Sigma-Aldrich) at $95^{\circ} \mathrm{C}$ for $10 \mathrm{~min}$. The slides were incubated with fresh $3 \%$ hydrogen peroxide (Sigma-Aldrich) for $10 \mathrm{~min}$ at room temperature and were washed in deionized water three times every $5 \mathrm{~min}$. The sections were then incubated with polyclonal primary antibodies against ionized calcium-binding adapter molecule 1 (Iba-1; rabbit anti-mouse; 1:1000; cat. no. , 019-19741; Wako, Carpinteria, CA, USA), neuronal nuclear antigen (NeuN; rabbit anti-mouse; 1:200; cat. no. ABN78A4; EMD Millipore, Billerica, MA, USA), or Beclin 1 (1:500; rabbit anti-mouse; cat. no. ab62557; Abcam, Cambridge, MA, USA) overnight at $4^{\circ} \mathrm{C}$. Following rinsing with PBS, the slides were incubated with horseradish peroxidase (HRP)-conjugated anti-rabbit immunoglobulin $\mathrm{G}$ ( $\mathrm{IgG}$ ) or anti-mouse $\mathrm{IgG}$ for $30 \mathrm{~min}$ at room temperature, followed by reacting with fresh diaminobenzidine solution (99\%; Sigma-Aldrich). The sections were hematoxylin-stained, dehydrated and mounted. Images were captured using a light microscope (Olympus BX51; Olympus) and immunofluorescence data were analyzed using
Image-Pro Plus (Media Cybernetics, Inc., Rockville, MD, USA).

Western blotting. Western blotting was performed, as described previously (19). Briefly, the cortical samples were homogenized and centrifuged at $14,000 \mathrm{x}$ g for $20 \mathrm{~min}$ at $4^{\circ} \mathrm{C}$. The resulting supernatants were centrifuged for $10 \mathrm{~min}$ and the protein content was measured using a bicinchoninic acid kit (Beyotime Institute of Biotechnology, Haimen, China). Equal quantities of protein $(60 \mu \mathrm{g})$ from each sample were resuspended in loading buffer, denatured at $100^{\circ} \mathrm{C}$ for $3-5 \mathrm{~min}$, separated using 10-12\% sodium dodecyl sulfate-polyacrylamide gel electrophoresis (Hangzhou Fu De Biological Technology Co., Ltd., Hangzhou, China) at a constant current and transferred onto polyvinylidene fluoride membranes (Hangzhou Fu De Biological Technology Co., Ltd). The membranes were blocked with non-fat dry milk for $2 \mathrm{~h}$ and were subsequently incubated overnight at $4^{\circ} \mathrm{C}$ with polyclonal primary antibodies against microtubule-associated protein light chain 3 (LC3; 1:1,000; rabbit ant-mouse; cat. no. 2775; Cell Signaling Technology, Inc., MA, USA), Beclin 1 (1:500; 


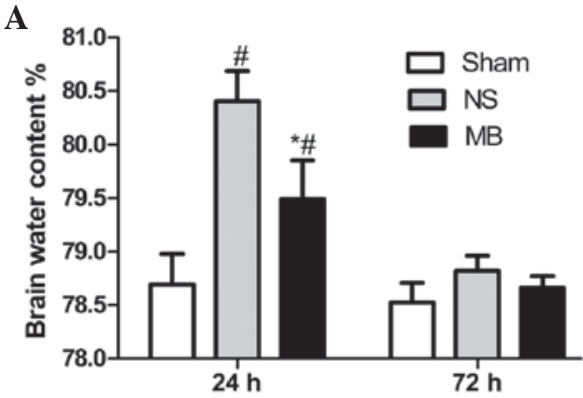

C

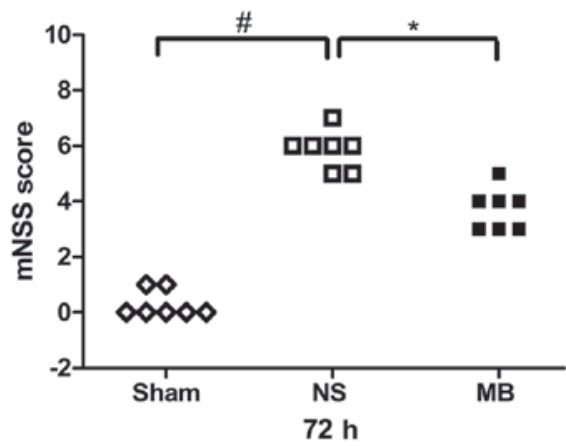

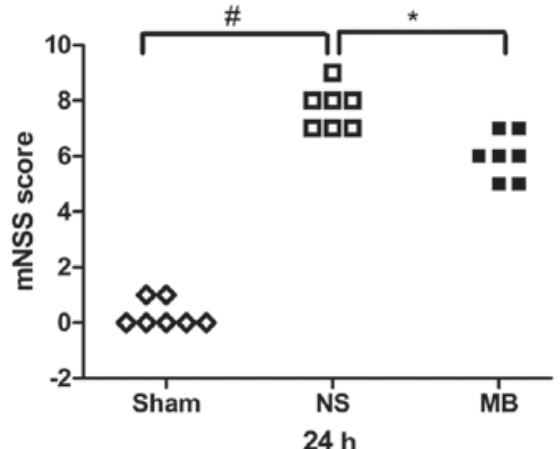

D

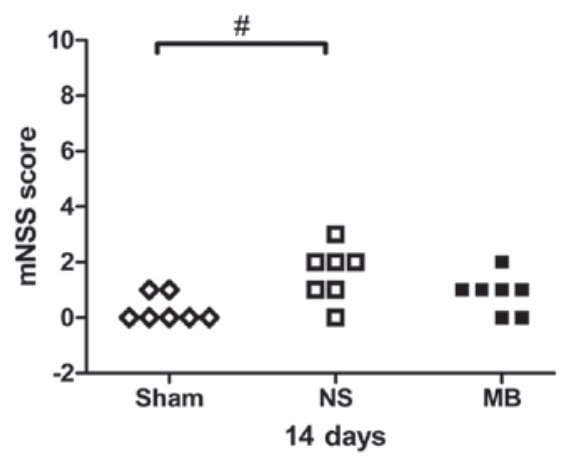

Figure 1. Effect of MB on brain edema and neurological function following TBI. (A) MB reduced BWC $24 \mathrm{~h}$ post-TBI. At $72 \mathrm{~h}$ post-TBI, the BWC decreased. Data are expressed as the mean \pm standard deviation $\left(n=6 ;{ }^{~} \mathrm{P}<0.05\right.$, vs. Sham). (B-D) MB significantly reduced the neurological deficits, compared with the vehicle group at 24 and $72 \mathrm{~h}$ post-TBI. ( $\mathrm{n}=7$; ${ }^{*} \mathrm{P}<0.05$, vs. Sham; ${ }^{*} \mathrm{P}<0.05$, vs. vehicle). MB, methylene blue; TBI, traumatic brain injury; BWC, brain water content; NS, normal saline; mNSS, modified neurological severity score.

rabbit ant-mouse; cat. no. ab63557; Abcam) and $\beta$-actin (1:2,000;rabbit ant-mouse; cat. no. sc-130656; Santa Cruz Biotechnology, Dallas, TX, USA). The membranes then were processed with HRP-conjugated secondary antibodies for $1 \mathrm{~h}$ at room temperature and protein bands were visualized on X-ray film (Kodak, Rochester, Y, USA) and quantified using ImageJ software 1.48U (National Institutes of Health, Bethesda, MA, USA).

Statistical analysis. All data are expressed as the mean \pm standard deviation and were analyzed using SPSS 13.0 statistical software (SPSS, Inc., Chicago, IL, USA). Two experimental groups were compared using Student's t-test and differences between the groups were analyzed using one-way analysis of variance, followed by the Student-Newman-Keuls test. $\mathrm{P}<0.05$ was considered to indicate a statistically significant difference.

\section{Results}

$M B$ alleviates cerebral edema. The injured hemisphere BWC was significantly increased $24 \mathrm{~h}$ post-TBI, whereas treatment with $\mathrm{MB}$ attenuated this increase (sham, 78.5\%; vehicle, $80.7 \%$; MB, 79.5\%; $\mathrm{P}<0.05$; Fig. 1A). However, the contralateral hemisphere, cerebellum and brain stem BWCs were not significantly different among the groups (data not shown). At $72 \mathrm{~h}$ post-TBI, the BWC decreased, however, no the vehicle $(78.9 \%)$ and $\mathrm{MB}(78.6 \%)$ groups demonstrated no statistically significant different $(\mathrm{P}>0.05$; Fig. 1A), which indicated that the CCI-induced cerebral edema began to subside $72 \mathrm{~h}$ post-TBI.
$M B$ reduces neurological functional deficits. TBI resulted in neurological functional deficits (Fig. 1B-D), determined using mNSS. Compared with the vehicle group, the mNSS scores were significantly lower in the MB group 24 and $72 \mathrm{~h}$ post-TBI, which indicated that MB significantly reduced the neurological deficits $(\mathrm{P}<0.05)$. However, no significant differences were identified in the scores between the two groups 14 days post-TBI $(\mathrm{P}>0.05)$, suggesting that functional compensation occurred in the two groups. Notably, a significant difference in neurological function remained between the vehicle and sham groups $(\mathrm{P}<0.05)$, which was not observed between the MB group and the sham group $(\mathrm{P}>0.05)$.

$M B$ reduces cerebral lesion volume and prevents neuronal death. The cerebral lesion volumes was assessed to confirm the neuroprotective effect of MB in TBI. The lesion volumes in the MB group lesion were significantly smaller, compared with those of the vehicle group at $24 \mathrm{~h}\left(2.49\right.$, vs, $\left.2.98 \mathrm{~mm}^{3}\right)$, $72 \mathrm{~h}\left(2.54\right.$, vs, $\left.3.29 \mathrm{~mm}^{3}\right)$ and 14 days $\left(1.44 \mathrm{vs}, 2.24 \mathrm{~mm}^{3}\right.$; $\mathrm{P}<0.05)$, as shown in Fig. 2). The NeuN staining confirmed the effect of MB in preventing neuronal death and revealed the presence of a significantly higher number of neurons in the MB group, compared with the vehicle group at $24 \mathrm{~h}$ $\left(1,014\right.$, vs, 815 cells $\left./ \mathrm{mm}^{2}\right)$ and $72 \mathrm{~h}\left(976\right.$, vs, 719 cells $/ \mathrm{mm}^{2}$; $\mathrm{P}<0.05$; Fig. 3A-C), which indicated that $\mathrm{MB}$ prevented neuronal death at the site of injury.

$M B$ inhibits microglial activation. Since neuroinflammation is important in the pathogenesis of TBI, the effect of $\mathrm{MB}$ on microglial activation was assessed using Iba-1 

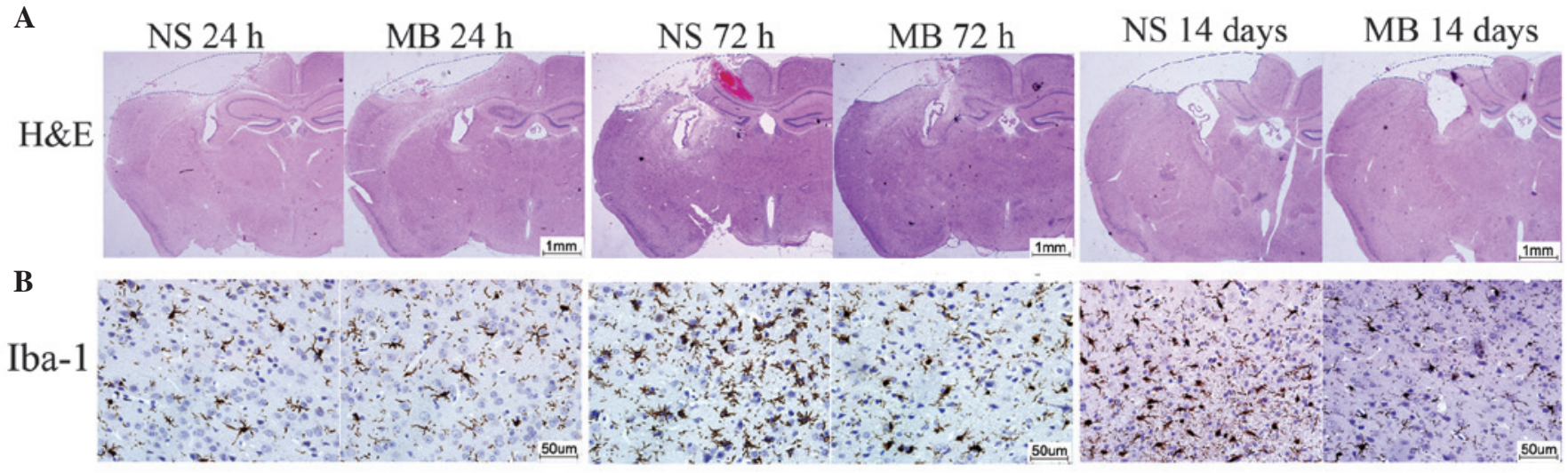

C

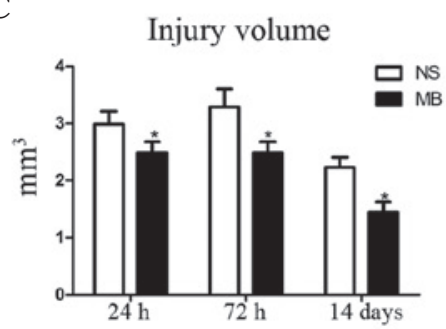

D

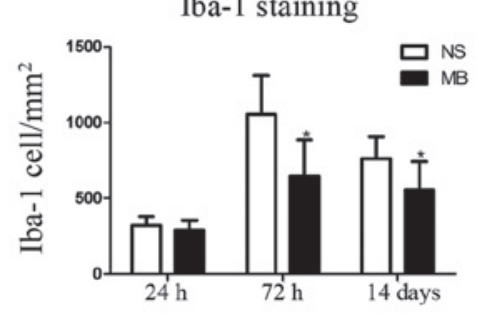

$\mathbf{E}$

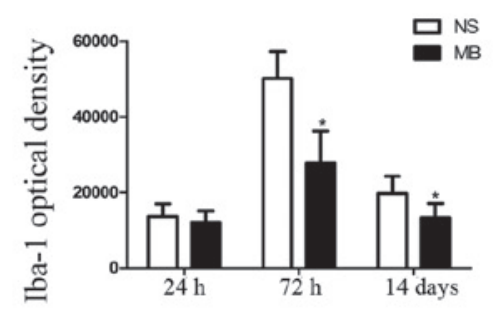

Figure 2. MB reduces cerebral lesion volume and microglial activation following TBI. (A) Representative images of HE-stained coronal sections 24 h, 72 $\mathrm{h}$ and 14 days post-TBI (Scale bar=1 mm). (B) Representative images of Iba-1-stained coronal sections $24 \mathrm{~h}, 72 \mathrm{~h}$ and $14 \mathrm{days}$ post-TBI (Scale bar=50 $\mu \mathrm{m})$. (C) Quantitative analysis of injury volume revealed a significant decrease in loss of tissue following treatment with $M B$ ( $\mathrm{n}=7$; * $\mathrm{P}<0.05$ ). (D and $\mathrm{E}$ ) Quantitative analysis demonstrated reduced Iba-1-positive cell numbers and fluorescence intensity following treatment with $\mathrm{MB}$. (n=6; $\left.{ }^{*} \mathrm{P}<0.05\right)$. The data are presented as the mean \pm standard deviation. MB, methylene blue; TBI, traumatic brain injury; NS, normal saline; HE, hematoxylin and eosin.

immunostaining. The number of microglia was low at $24 \mathrm{~h}$ post-TBI, however, activation was observed at $72 \mathrm{~h}$, which persisted until 14 days post-TBI (Fig. 2). Although the numbers of Iba-1-positive cells in the MB and vehicle groups at $24 \mathrm{~h}$ post-TBI were not significantly different (292, vs, 320 cells $/ \mathrm{mm}^{2}$; P>0.05; Fig. 2), marked inhibition of microglial activation was observed in the MB-treated mice, compared with the vehicle mice at $72 \mathrm{~h}(644, \mathrm{vs}, 1,053)$ and 14 days ((557, vs, 762 cells $\left./ \mathrm{mm}^{2}\right)$ post-TBI (P<0.05; Fig. 2$)$. The analysis using ImagePro Plus for identification of the Iba-1 optical densities of the two groups yielded similar

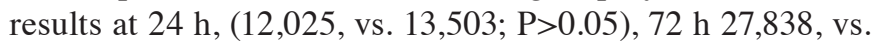
$50,107 ; \mathrm{P}<0.05)$ and at 14 days $(13,202$, vs. 19,$725 ; \mathrm{P}<0.05$; Fig. 2) following TBI.

$M B$ increases autophagy. MB promotes autophagy in ex vivo slice cultures or primary neurons (20). To examine the possible mechanism underlying the action of MB against TBI, the effect of MB on autophagy was assessed using Beclin 1 immunostaining. Intense Beclin 1 staining was observed in the cytoplasm, which was more prominent in the MB group (Fig. 3D and E), compared with the vehicle group. Based on the increased expression of Beclin 1 and increased LC3-II/LC3-I ratio, the level of autophagy was higher in the MB group, compared with the vehicle group at $24 \mathrm{~h}$ (Beclin 1, 4.4, vs, 3.2-fold; LC3-II/I, 1.29, vs. 0.63) and $72 \mathrm{~h}$ (Beclin 1, 2.8, vs, 2.0-fold; LC3-II/I, 1.06, vs. 0.56) post-TBI $(\mathrm{P}<0.05$; Fig. 4A-F). This suggested that MB increased autophagy, resulting in high levels of autophagic traffic in the acute phase of TBI.

\section{Discussion}

TBI is a major cause of mortality worldwide, and survivors may suffer permanent neurological impairment, including reduced cognitive function (21), neurodegenerative disorders, including Alzheimer's disease (22), and brain tumors (23). TBI can be divided into primary and secondary injury. Primary injury mechanisms result from initial mechanical damage, leading to neuronal, axonal and glial death, and blood vessel breakage (24). The long-term consequences of TBI predominantly result from the mechanisms of secondary injury (25), in cases where the primary tissue damage cannot be treated. Therefore, inhibiting or reducing secondary brain injury is key in the treatment of TBI for improving injury outcomes. The results of the present study demonstrated that $\mathrm{MB}$ attenuated TBI-induced edema, neuroinflammation and neuronal death, increased autophagy and reduced cerebral lesion volume.

In the present study, treatment with $1.5 \mathrm{mg} / \mathrm{kg}$ intraperitoneal MB on the first day of injury attenuated the TBI-induced increase in the BWC of the injured hemisphere. It is important to note that TBI-associated mortality is generally caused by cerebral edema and increased intracranial pressure (26). In the present study, the BWC of the MB group was significantly decreased $24 \mathrm{~h}$ post-TBI, although the differences between the $\mathrm{MB}$ and vehicle groups were not significant. However, even a small increase in BWC can lead to significantly increased intracranial pressure and poorer outcomes (27). These data indicated that MB may improve neurological outcome and survival rates following moderate to severe TBI. Post-traumatic brain edema is considered to be generated by vasogenic edema 
A

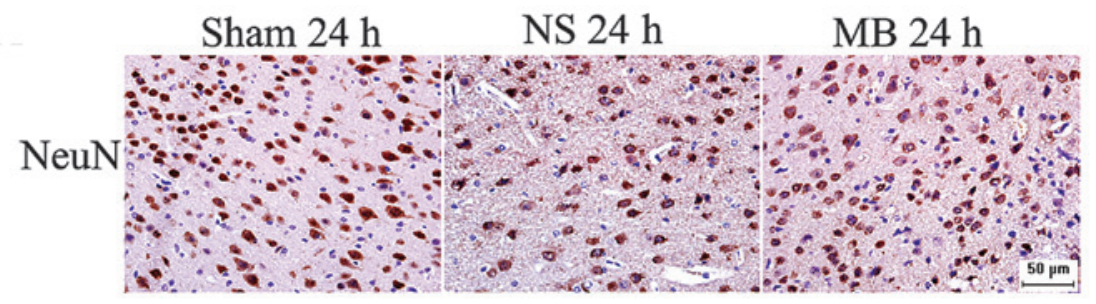

B

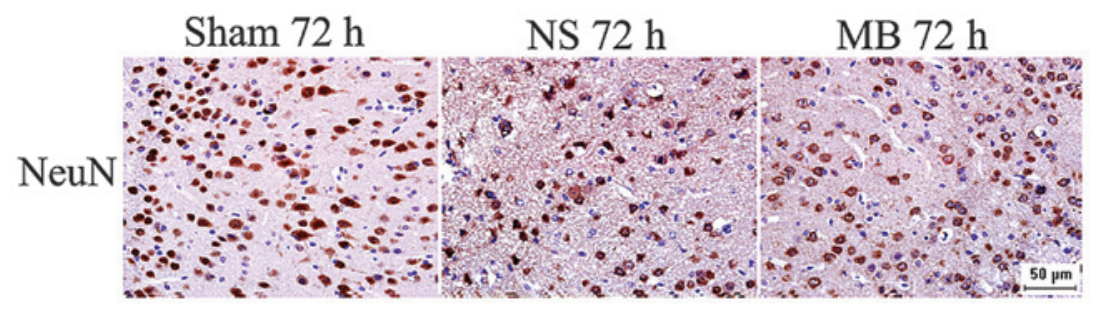

C
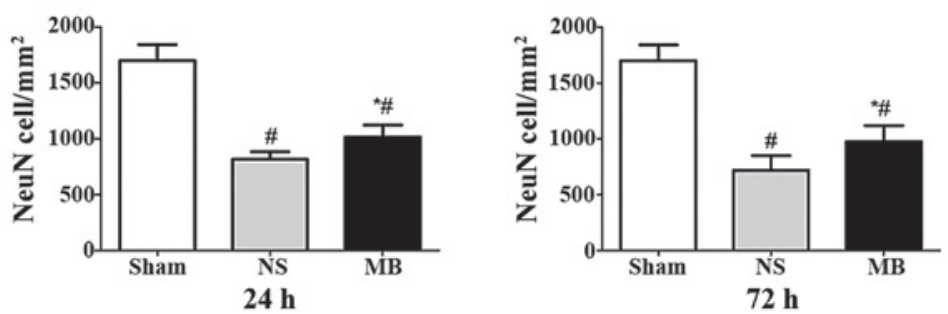

D

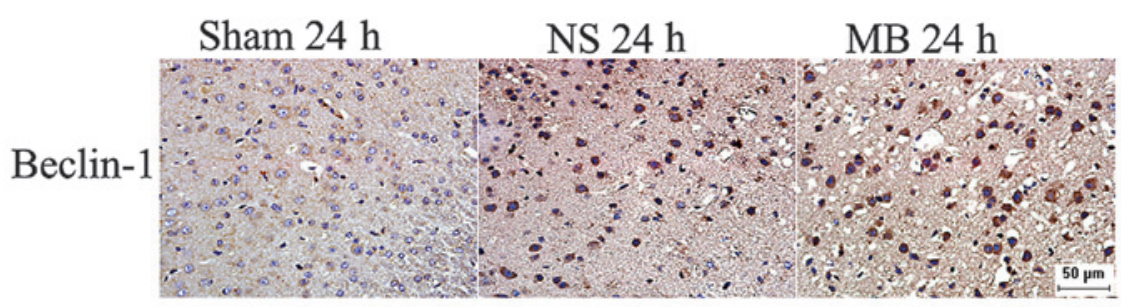

$\mathbf{E}$

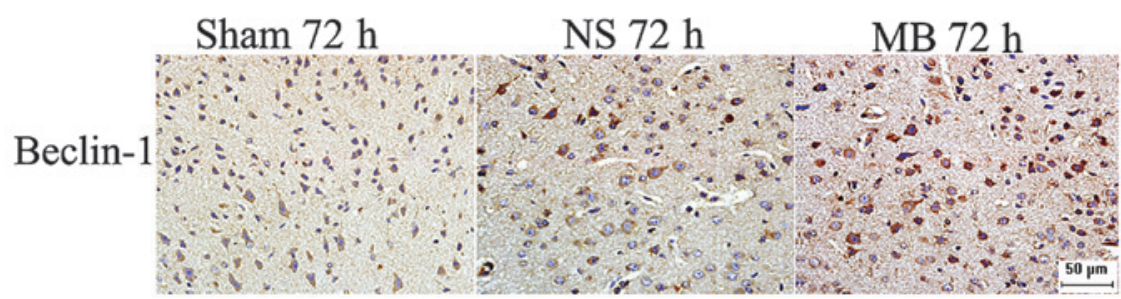

Figure 3. Methylene blue prevents neuronal death and promotes autophagy at the perilesional area following TBI. (A and B) Representative images of NeuN-stained coronal tissue sections 24 and $72 \mathrm{~h}$ post-TBI. (Scale bar=50 $\mu \mathrm{m}$ ). (C) Quantitative analysis revealed a significant increase in the number of NeuN-positive cells 24 and $72 \mathrm{~h}$ post-TBI following treatment with MB. The data are presented as the mean \pm standard deviaton $\left(\mathrm{n}=6\right.$; $\left.{ }^{*} \mathrm{P}<0.05\right)$. (D and E) Representative images of Beclin 1-stained coronal tissue sections 24 and $72 \mathrm{~h}$ post-TBI demonstrating marked cytoplasmic staining in the MB-treated cells (Scale bar=50 $\mu \mathrm{m}$ ). MB, methylene blue; TBI, traumatic brain injury; NS, normal saline.

and cytotoxic edema (28). The former commonly occurs within a few hours following injury, followed by the latter, which may persist for weeks (29). No statistical difference was observed in the BWC between the two groups $72 \mathrm{~h}$ post-injury, which indicated that MB may have reduced vasogenic edema in the CCI model. However, the specific mechanisms of $\mathrm{MB}$ in decreasing cerebral edema require further investigation.

TBI initiates a neuroinflammatory cascade, which is characterized by microglial activation and increased production of proinflammatory cytokines (30). Microglia release neurotrophic factors, including nerve growth factor, brain-derived neurotrophic factor and neurotrophin-3, which promote neuronal growth and survival (31). The detrimental effects of prolonged microglial activation, accompanied by a neuroinflammatory cascade, may counter these benefits (32). Microglial activation persists for several years following the initiating TBI, in which persistent microglial activation is correlated with the degree of cognitive impairment (33) and may predispose to the development of neurodegenerative disorders, including Alzheimer's disease (32). Nitric oxide (NO), a signaling molecule that freely diffuses across the plasmalemma and hundreds of micrometers between cells, is generated via enzymatic L-arginine cleavage by NO synthase (NOS). By binding to the heme group of soluble guanylate cyclase (sGC), NO increases the conversion of 
A

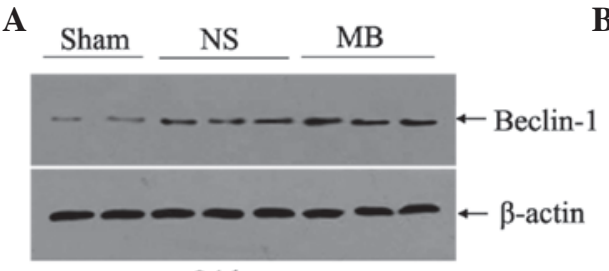

D

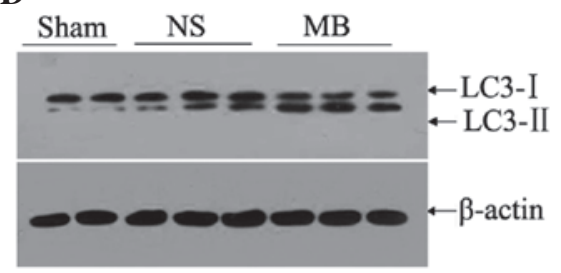

$24 \mathrm{~h}$

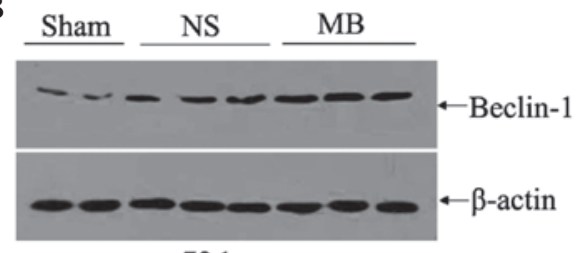

$72 \mathrm{~h}$

E

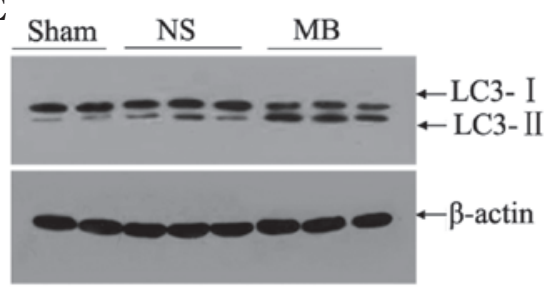

C

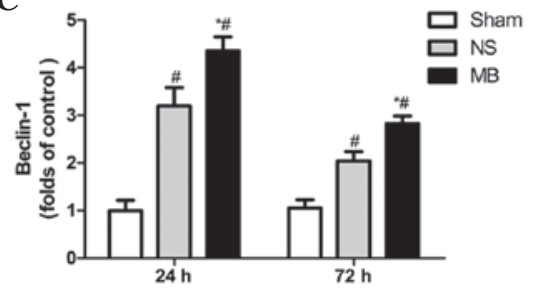

F

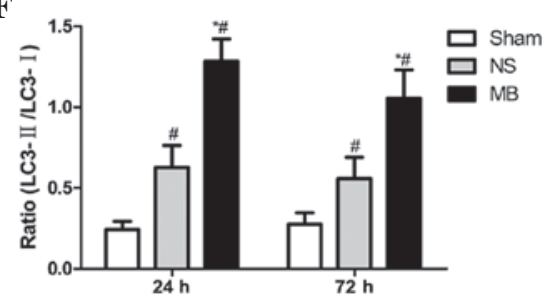

Figure 4. Effect of MB on autophagy, determined using western blot analysis. (A-D) Representative western blot analyses of expression levels of Beclin 1 and LC3 in the ipsilateral cortex 24 and $72 \mathrm{~h}$ post-TBI. (E and F) Relative band densities of LC3 and Beclin 1 were determined 24 and 72 h post-TBI. MB significantly upregulated the expression of Beclin 1 and increased the LC3-II/LC3-I ratio, compared with the vehicle control group. The protein band densities were analyzed and normalized against $\beta$-actin. Lanes 1 and 2, sham group; lanes 3-5, vehicle group; lanes 6-8, MB group. The data are expressed as a percentage of the sham group and are presented as the mean \pm standard deviation $\left(\mathrm{n}=6\right.$; ${ }^{\#} \mathrm{P}<0.05$, vs. sham; ${ }^{*} \mathrm{P}<0.05$, vs. vehicle). MB, methylene blue; TBI, traumatic brain injury; NS, normal saline.

guanidine triphosphate to cyclic guanidine monophosphate (cGMP), acting as an early chemoattractant and microglial activator $(34,35)$. MB eliminates cGMP immunoreactivity at lesions and inhibits microglial accumulation (34). Other possible mechanisms of MB in microglial inhibition include binding to peroxynitrites, to NO itself, or inhibiting NOS activity (36). Using a superoxide dismutase 1 gene mutation G93A (SOD1G93A) model of amyotrophic lateral sclerosis, Dibaj et al (10) demonstrated that MB inhibits the microglial reaction in control and in SOD1G93A mice. However, compared with the present study, no alterations in microglial activity were observed in the systemically MB-treated mice, compared with the controls. In the present study, microglial activation was observed following TBI, which peaked at $73 \mathrm{~h}$ and persisted until 14 days post-TBI. Intraperitoneal MB significantly inhibited the microglial activation at $72 \mathrm{~h}$ and 14 days post-TBI. In addition to the model and experimental design differences, the different usage of MB may explain the contradictory results.

Autophagy degrades long-lived stable proteins, and entire organelles can be recycled under stress conditions or during development (37). Autophagy is important for homeostasis and serves as a cellular mechanism for eliminating destructive organelles and recycling components for reuse. Diskin et al (38) first reported in 2005, that TBI may stimulate autophagy. Using a mouse model of closed head injury, Beclin 1, required for initiating autophagy, was observed to increase in the neurons and activated astrocytes at the site of injury, providing indirect evidence that autophagy is increased following TBI. The effects of rapamycin, an inducer of autophagy, has been assessed in the same model, revealing that the increase in autophagy produced beneficial neurological effects and increased the numbers of surviving neurons at the site of injury (39). In the present study, upregulation of Beclin 1 and an increased LC3-II/LC3-I ratio was observed 24 and $72 \mathrm{~h}$ post-TBI in the MB- and vehicle-treated mice, indicating the activation of autophagy. However, the difference between the two groups was statistically significant, as were the number of surviving neuron numbers in the MB group. These data indicated that $\mathrm{MB}$ intervention promoted autophagy and may assist in preventing neuronal death following TBI. To date, few studies have focused on the association between MB and autophagy. Using cell models and organotypic brain slice cultures from a mouse model of frontotemporal dementia, Congdon et al (20) confirmed that MB alters the expression levels of LC3-II, Beclin 1 and p62, suggesting that MB is a potent inducer of autophagy. Wang et al (40) reported that MB inhibits the activity of heat shock protein 70 prior to promoting autophagy. The present study demonstrated that MB promotedautophagy in the CCI model of TBI. This is the first report, to the best of our knowledge, to demonstrate the effect of MB on autophagy in TBI, however, the specific mechanism remains to be elucidated.

In conclusion, the present study demonstrated that $\mathrm{MB}$ exerted a neuroprotective effect by increasing autophagy, decreasing brain edema and inhibiting microglial activation, which reduced cerebral lesion volume, neuronal death and neurological deficit. Notably, the US FDA has approved MB as a drug (12) and, at low doses, no side effects have been identified (9). The present study did not examine the molecular mechanisms underlying the effects of $\mathrm{MB}$ in the regulation of autophagy and microglial activation, and further investigation of these possible mechanisms is required. In addition, investigations to determine a suitable dose and application duration, and to determine the side effects of MB in treatment of TBI is required.

\section{Acknowledgements}

This study was supported by a grant from the National Natural Science Foundation of China (grant. no. 81200956). This manuscript was copyedited by an English speaking professional with a science background at Elixigen Corporation (Huntington Beach, CA, USA). 


\section{References}

1. Feigin VL, Theadom A, Barker-Collo S, Starkey NJ, McPherson K, Kahan M, Dowell A, Brown P, Parag V, Kydd R, et al; BIONIC Study Group: Incidence of traumatic brain injury in New Zealand: A population-based study. Lancet Neurol 12: 53-64, 2013.

2. Dvela-Levitt M1, Ami HC, Rosen H, Shohami E and Lichtstein D: Ouabain improves functional recovery following traumatic brain injury. J Neurotrauma 31: 1942-1947, 2014

3. Fenn AM, Skendelas JP, Moussa DN, Muccigrosso MM, Popovich PG, Lifshitz J, Eiferman DS and Godbout JP: Methylene blue attenuates traumatic brain injury-associated neuroinflammation and acute depressive-like behavior in mice. J Neurotrauma 32: 127-138, 2014

4. Xiong Y, Mahmood A and Chopp M: Animal models of traumatic brain injury. Nat Rev Neurosci 14: 128-142, 2013.

5. Auchter A, Williams J, Barksdale B, Monfils MH and Gonzalez-Lima F: Therapeutic benefits of methylene blue on cognitive impairment during chronic cerebral hypoperfusion. J Alzheimers Dis 42 Suppl 4: S525-S535, 2014.

6. Kwok ES and Howes D: Use of methylene blue in sepsis: A systematic review. J Intensive Care Med 21: 359-363, 2006.

7. Maslow AD, Stearns G, Butala P, Schwartz CS, Gough J and Singh AK: The hemodynamic effects of methylene blue when administered at the onset of cardiopulmonary bypass. Anesth Analg 103: 2-8, 2006.

8. Paciullo CA, McMahon Horner D, Hatton KW and Flynn JD: Methylene blue for the treatment of septic shock. Pharmacotherapy 30: 702-715, 2010.

9. Rojas JC, Bruchey AK and Gonzalez-Lima F: Neurometabolic mechanisms for memory enhancement and neuroprotection of methylene blue. Prog Neurobiol 96: 32-45, 2012.

10. Dibaj P, Zschüntzsch J, Steffens H, Scheffel J, Göricke B, Weishaupt JH, Le Meur K, Kirchhoff F, Hanisch UK, Schomburg ED, et al: Influence of methylene blue on microglia-induced inflammation and motor neuron degeneration in the SOD1(G93A) model for ALS. PLoS One 7 : e43963, 2012.

11. Shen Q, Du F, Huang S, Rodriguez P, Watts LT and Duong TQ Neuroprotective efficacy of methylene blue in ischemic stroke: An MRI study. PLoS One 8: e79833, 2013

12. Talley Watts L, Long JA, Chemello J, Van Koughnet S, Fernandez A, Huang S, Shen Q and Duong TQ: Methylene blue is neuroprotective against mild traumatic brain injury. J Neurotrauma 31: 1063-1071, 2014.

13. National Research Council (US) Committee for the Update of the Guide for the Care and Use of Laboratory Animals: Guide for the Care and Use of Laboratory Animals. 8th edition. National Academies Press, Washington DC, 2011

14. Hong Y, Yan W, Chen S, Sun CR and Zhang JM: The role of Nrf2 signaling in the regulation of antioxidants and detoxifying enzymes after traumatic brain injury in rats and mice. Acta Pharmacol Sin 31: 1421-1430, 2010.

15. Callaway NL, Riha PD, Bruchey AK, Munshi Z and Gonzalez-Lima F: Methylene blue improves brain oxidative metabolism and memory retention in rats. Pharmacol Biochem Behav 77: 175-181, 2004

16. Chen J, Sanberg PR, Li Y, Wang L, Lu M, Willing AE, Sanchez-Ramos J and Chopp M: Intravenous administration of human umbilical cord blood reduces behavioral deficits after stroke in rats. Stroke 32: 2682-2688, 2001.

17. Xu J, Wang H, Ding K, Lu X, Li T, Wang J, Wang $\mathrm{C}$ and Wang J: Inhibition of cathepsin $\mathrm{S}$ produces neuroprotective effects after traumatic brain injury in mice. Mediators Inflamm 2013: 187873,2013

18. Wang H, Liao Z, Sun X, Shi Q, Huo G, Xie Y, Tang X, Zhi $X$ and Tang $Z$ : Intravenous administration of Honokiol provides neuroprotection and improves functional recovery after traumatic brain injury through cell cycle inhibition Neuropharmacology 86: 9-21, 2014

19. Chen J, Wang L, Wu C, Hu Q, Gu C, Yan F, Li J, Yan W and Chen G: Melatonin-enhanced autophagy protects against neural apoptosis via a mitochondrial pathway in early brain injury following a subarachnoid hemorrhage. J Pineal Res 56 $12-19,2014$
20. Congdon EE, Wu JW, Myeku N, Figueroa YH, Herman M, Marinec PS, Gestwicki JE, Dickey CA, Yu WH and Duff KE: Methylthioninium chloride (methylene blue) induces autophagy and attenuates tauopathy in vitro and in vivo. Autophagy 8: 609-622, 2012

21. Humphreys I, Wood RL, Phillips CJ and Macey S: The costs of traumatic brain injury: A literature review. Clinicoecon Outcomes Res 5: 281-287, 2013

22. Sivanandam TM and Thakur MK: Traumatic brain injury: A risk factor for Alzheimer's disease. Neurosci Biobehav Rev 36: 1376-1381, 2012

23. Han Z, Du Y, Qi H and Yin W: Post-traumatic malignant glioma in a pregnant woman: Case report and review of the literature. Neurol Med Chir (Tokyo) 53: 630-634, 2013.

24. Kumar A and Loane DJ: Neuroinflammation after traumatic brain injury: Opportunities for therapeutic intervention. Brain Behav Immun 26: 1191-1201, 2012.

25. Eroglu B, Kimbler DE, Pang J, Choi J, Moskophidis D, Yanasak N, Dhandapani KM and Mivechi NF. Therapeutic inducers of the HSP70/HSP110 protect mice against traumatic brain injury. J Neurochem 130: 626-641, 2014.

26. Lu J, Marmarou A, Choi S, Maas A, Murray G and Steyerberg EW; Impact and Abic Study Group: Mortality from traumatic brain injury. Acta Neurochir Suppl (Wien) 95: 281-285, 2005.

27. Xu FF, Sun S, Ho AS, Lee D, Kiang KM, Zhang XQ, Wang AM, Wu EX, Lui WM, Liu BY and Leung GK: Effects of progesterone vs. dexamethasone on brain oedema and inflammatory responses following experimental brain resection. Brain Inj 28: 1594-1601, 2014.

28. Esen F, Erdem T, Aktan D, Kalayci R, Cakar N, Kaya M and Telci L: Effects of magnesium administration on brain edema and blood-brain barrier breakdown after experimental traumatic brain injury in rats. J Neurosurg Anesthesiol 15: 119-125, 2003.

29. Barzó P, Marmarou A, Fatouros P, Hayasaki K and Corwin F: Contribution of vasogenic and cellular edema to traumatic brain swelling measured by diffusion-weighted imaging. J Neurosurg 87: 900-907, 1997.

30. Lim SW, Wang CC, Wang YH, Chio CC, Niu KC and Kuo JR: Microglial activation induced by traumatic brain injury is suppressed by postinjury treatment with hyperbaric oxygen therapy. J Surg Res 184: 1076-1084, 2013.

31. Parekkadan B, Berdichevsky Y, Irimia D, Leeder A, Yarmush G, Toner M, Levine JB and Yarmush ML: Cell-cell interaction modulates neuroectodermal specification of embryonic stem cells. Neurosci Lett 438: 190-195, 2008.

32. Gentleman SM, Leclercq PD, Moyes L, Graham DI, Smith C, Griffin WS and Nicoll JA: Long-term intracerebral inflammatory response after traumatic brain injury. Forensic Sci Int 146: 97-104, 2004.

33. Ramlackhansingh AF, Brooks DJ, Greenwood RJ, Bose SK, Turkheimer FE, Kinnunen KM, Gentleman S, Heckemann RA, Gunanayagam K, Gelosa $\mathrm{G}$, et al: Inflammation after trauma: Microglial activation and traumatic brain injury. Ann Neurol 70: 374-383, 2011.

34. Duan Y, Haugabook SJ, Sahley CL and Muller KJ: Methylene blue blocks cGMP production and disrupts directed migration of microglia to nerve lesions in the leech CNS. J Neurobiol 57: 183-192, 2003.

35. Dibaj P,Nadrigny F, Steffens H, Scheller A, Hirrlinger J, Schomburg ED, Neusch C and Kirchhoff F: NO mediates microglial response to acute spinal cord injury under ATP control in vivo. Glia 58: 1133-1144, 2010.

36. Gibbs SM and Truman JW: Nitric oxide and cyclic GMP regulate retinal patterning in the optic lobe of Drosophila. Neuron 20: 83-93, 1998.

37. Levine B and Klionsky DJ: Development by self-digestion: Molecular mechanisms and biological functions of autophagy. Dev Cell 6: 463-477, 2004.

38. Diskin T, Tal-Or P, Erlich S, Mizrachy L, Alexandrovich A, Shohami E and Pinkas-Kramarski R: Closed head injury induces upregulation of Beclin 1 at the cortical site of injury. J Neurotrauma 22: 750-762, 2005.

39. Erlich S, Alexandrovich A, Shohami E and Pinkas-Kramarski R: Rapamycin is a neuroprotective treatment for traumatic brain injury. Neurobiol Dis 26: 86-93, 2007.

40. Wang AM, Morishima Y, Clapp KM, Peng HM, Pratt WB, Gestwicki JE, Osawa Y and Lieberman AP: Inhibition of hsp70 by methylene blue affects signaling protein function and ubiquitination and modulates polyglutamine protein degradation. J Biol Chem 285: 15714-15723, 2010 